cability to the study of fundamental biological questions, by providing a direct means to study coupling between biochemical and biomechanical reaction cycles."

STEVEN TROHALAKI

\section{High-Velocity Ballistic Impact with Boron Carbide Produces Localized Amorphization}

Localized amorphization induced by shock has been observed in crystalline boron carbide. Mingwei Chen and Kevin Hemker of Johns Hopkins University and James McCauley of the U.S. Army Research Laboratory at Aberdeen Proving Ground have demonstrated that extremely high-rate deformation can initiate formation of amorphized bands in the rhombohedral crystal structure of $\mathrm{B}_{4} \mathrm{C}$.

Boron carbide is commonly used as ballistic armor. Although the material is effective for protection against low-energy projectiles such as bullets from handguns, it is less effective upon more powerful impacts. As reported in the March 7 issue of Science, Chen, McCauley, and Hemker found that the measured drop in the impact resistance of boron carbide at impact pressures in the range of $20-23 \mathrm{GPa}$ could be attributed to the formation of nanoscale intragranular amorphous bands in the crystal.

The researchers observed the amorphous bands by high-resolution electron microscopy (HREM). They used transmission electron microscope electron energy-loss spectroscopy to compare the chemical composition of the amorphous regions to that of the crystalline $B_{4} C$. These spectra allowed the researchers to rule out the possibility of a pressureinduced decomposition or chemical reaction since there was no detectable difference in chemical composition. The researchers also determined the HREM images were not consistent with rebonding of two cracked surfaces or melting. Accordingly, they believe that the amorphization is a solid-state transformation instigated by the ballistic event.

Recent diamond anvil studies demonstrated a phase transition at a pressure around $20 \mathrm{GPa}$, and previous nanoindentation experiments at even higher pressures suggested amorphization, but this speculation was discounted based on Raman spectra. The researchers in this study suggest that their results warrant renewed scrutiny of the previous interpretations of the diamond anvil and nanoindentation results.

The drop in performance of boron carbide when exposed to high-impact pressures has been known for years. The cur- rent research provides a microscopic explanation for this highly unfavorable property for a material employed as ballistic armor. The researchers do not propose a means to avoid formation of the amorphization regions that appear to be responsible for the drop in strength of crystalline boron carbide. Nevertheless, they speculate that this improved understanding of how shock can alter materials properties and permit the synthesis of novel structures could further expand the realm of possibilities for innovative materials synthesis.

Chen said, "It's like having a sturdy table and suddenly kicking the legs out from underneath it."

"This discovery was very enlightening, because it tells us that under extremely high pressures the crystal structure collapses and forms these nanoscale amorphous bands," said Hemker. "Then the material fractures along these bands because the glassy material appears to be weaker than the crystalline boron carbide."

McCauley said, "The question now is, how should we try to change the boron carbide? We intend to try modifying the material's grain structure, its chemistry, and the additives used in making it. The goal will be to have the amorphization occur at higher impact pressures. Then the armor would provide better protection against a wider range of threats."

Although the findings have immediate implications in the production of improved armor materials, the researchers said that their observations also provide experimental evidence that extreme conditions in pressure, temperature, and/or loading and quenching rates can lead to the creation of entirely new materials or structures with substantially altered physical and mechanical properties.

"We have a new appreciation for how localized loss of crystalline structure can substantially alter materials properties," Hemker said. "I anticipate this understanding will allow scientists and engineers to tailor boron carbide's properties in a way that has not been exploited to date. We do not need to rely on chemical modifications or phase transformations to pursue novel materials properties. I hope our research will help to motivate exciting new avenues in materials design."

EMILY JARVIS

\section{Carbon Nanofibers with Smaller Diameter Rather than Larger Promote More Osteoblast Functions}

Nanomaterials in the form of ceramics, polymers, metals, and carbon nanofibers have recently been shown to improve bone, vascular, bladder, cartilage, and neural cell function. Doctoral student Rachel Price, under the direction of Thomas J. Webster in the Biomedical Engineering Department at Purdue University, has demonstrated that smallerdiameter carbon nanofibers promote more functions of the osteoblast, the boneforming cell, than do nanofibers with larger diameters. Such a conclusion was based on evidence that a statistically larger number of osteoblasts adhered, proliferated, and deposited calcium-containing material on the smaller-diameter (60-nm) carbonfiber compacts than to the larger-diameter (125-nm) carbon-fiber compacts, or to either a cobalt chromium or a titanium alloy. The latter two materials are currently used in dental and orthopedic applications.

As reported in the May issue of Biomaterials (24:11), there was a $33 \%$ increase in the number of adherent osteoblasts on the smaller carbon fibers than the larger and up to an $800 \%$ increase over the titanium alloy. In addition, in a carbon nanofiber/polycarbonate urethane composite, osteoblast adhesion increased on those composites when higher ratios of carbon nanofibers were incorporated.

Price said, "Another interesting finding is that competitive cell functions are adversely affected on the smaller carbon fibers over the same time span. Increased osteoblast function and decreased competitive cell functions imply that better bonding to juxtaposed bone can be achieved through the use of carbon nanofibers."

\section{Novel Method for Control of Quantum Entanglement Developed}

Entanglement refers to a quantum phenomenon whereby measurements performed on one particle affect another particle that is far away. Quantum entanglement can be exploited in quantum information applications such as cryptography and quantum computation. A recent report by Roberto Merlin and coworkers in the Departments of Physics at the University of Michigan, Ann Arbor, and the University of Notre Dame describes the controlled generation of entangled states involving the spin of $\mathrm{Mn}^{2+}$ and donor impurities in CdTe quantum wells. Their procedure relies on photoexcited excitons to mediate the interaction between spins. The method allows a previously unavailable level of control of particle entanglement. According to Merlin, "the procedure is potentially set-specific and scalable, thereby holding promise for quantum computing applications."

As described in the March issue of 